\title{
INTER-APPLICATION COMMUNICATION: A PRototype IMPLEMENTATION
}

\author{
Kalaiselvi Arunachalam ${ }^{1}$, Gopinath Ganapathy ${ }^{2}$ \\ ${ }^{1}$ School of Computer Science, Engineering and Applications, \\ Bharathidasan University, India \\ ${ }^{2}$ Registrar, Bharathidasan University, India
}

\begin{abstract}
A growing popularity of smart devices of various type, shape and form factor with multitude of applications from diverse categories and data are used to meet the demands of users in their digitally enriched living environment. The data sharing between these applications would be beneficial to the users when these heterogeneous devices are used together by them in their home network. The inter-application communication enables an application to discover, connect and share data with other applications across heterogeneous devices in a home network. This paper provides a prototype implementation of the inter-application communication in a home network along with a brief summary about its demand in near future.
\end{abstract}

\section{KEYWORDS}

Inter-application Communication, Prototype Implementation, Home Network, App-to-App Communication, Heterogeneous Devices

\section{INTRODUCTION}

The popularity of smart devices of various type, shape, form factor and the heterogeneity of applications on these devices with multitude of data like text, image, audio, video, graphics, hyperlink etc. to serve the users around the world in their digital living environment. It would be effective and beneficial to the user to share data directly between these heterogeneous applications across smart devices without any additional requirement of intermediary hardware or software. This paper provides a prototype implementation of inter-application communication in a home network in which two applications that reside on two different device types and operating systems can discover, connect and share data with each other. The inter-application communication would enable data sharing among heterogeneous applications by limiting additional installation, configuration, upgrade, porting, migration etc.

\section{RELATED WORKS}

The inter-application communication (IAC) promotes the development of feature rich applications that are discoverable, data sharable, compatible and interoperable together in a network. Some inter-application communication mechanisms were proposed earlier as below but they are limited with particular aspects.

A primary application uses an unique identifier for its identification and a random number for certifying the responsive communication received by it as in [1] where by the recipient application validates the unique identifier of the primary application and if it is valid, then it generates a second random number for certifying the subsequent communication submitted to it Dhinaharan Nagamalai et al. (Eds) : COMIT, AISCA - 2019

pp. 27 -36, 2019. () CS \& IT-CSCP 2019

DOI: $10.5121 /$ csit.2019.90103 
as in [1] but this system requires a mobile device to provide common platform, processor and memory for the communication between the applications. Another mechanism in which a system that automatically generates an interface code which provides a single comprehensive adaptation interface that integrates multiple executable applications which can be plugged in to the adaptation interface to support IAC as in [2] but it would be tedious to provide an interface code for different platforms. A proposed framework to develop portable software application that support a single design for deployment on multiple target platforms for mobile applications as in [3] and even though this framework conducts portability check on the devices, there is no feature for compatibility check on applications towards the interoperability among them. As in [4], the proposed system in which two applications are involved to share the authorization details to login to a resource server to access the network resources and the IAC is limited here to be used for single-sign on mechanism only. An audio based data sharing between mobile applications on two devices is proposed as in [5] in which data is transferred in the form of audio messages which are communicated over a speaker and microphone that are interfaced to the mobile devices but this mechanism requires very close proximity between the devices for communication and sufficient volume for the audio messages as well.

A data sharing system proposed in which multiple applications can share data between them as in [6] using a data sharing zone that shares the data based on the access policy by using the access rights assigned by the applications but the sharing zone can be only accessed within a device and it is not accessible by applications on other devices. The sharing of data between smart devices in a network as in [7] defines the sharing of an image across various smart devices in a network and this system is device-specific only and not application-specific. A shared storage location is used by a system in which the first mobile application uses a shared encryption key to encrypt the data to be transferred from it and a second mobile application is configured to retrieve the encrypted data from this shared storage location as in [8] but the shared storage location can be accessed only by the applications within a device. A proposed system that defines sharing of data across multiple electronic devices as in [9] in which sharing of data between devices carried out through a file sharing session and the data transfer across applications residing on these devices is limited in this system.

\section{SMart Devices AND APPliCATions}

A smart device is an electronic device that can connect and share data with other devices in a network through Ethernet, Wi-Fi, Bluetooth, NFC etc. There are several types of smart devices like Smartphone, Tablet, Notebook, Smart TV, Smart watch, Smart band, Smart key chain, Smart glass etc. as in [10]. There are billions of smart devices used by the people around the world and millions of applications as in [Table 1] from various categories are available in the popular application stores like Google Play as in [11], App Store as in [12], Windows Store [13], BlackBerry World as in [14] for the users. These applications are available for each mobile operating system separately on these stores and are optimized for the devices of different types and sizes for each mobile operating system. Some of the popular application categories that are available on all popular application stores are Books, Business, Education, Entertainment, Finance, Games, Health \& Fitness, Lifestyle, Magazines \& News, Maps \& Navigation, Music \& Audio, Photo \& Video, Productivity, Shopping, Social Networking, Sports, Travel and Utilities as in [11-14]. These applications involve various types of data like text, number, date, time, image, graphics, audio, video etc. which are required by the users in their daily life. 
Table 1. Popular App Stores vs. Applications

\begin{tabular}{|c|c|c|}
\hline App Store Name & Operating System & $\begin{array}{c}\text { Total Apps } \\
\text { (as of Dec. 2018) }\end{array}$ \\
\hline Google Play & Android & $2,100,000$ \\
\hline App Store & iOS & $2,000,000$ \\
\hline Windows Store & Microsoft Windows & 669,000 \\
\hline BlackBerry World & BlackBerry OS & 234,500 \\
\hline Amazon App Store & Android, BlackBerry & 450,000 \\
\hline
\end{tabular}

The number of applications available on these application stores are increasing every day as in [15] and hundreds of applications are added to these stores each day by the developers around the world. Due to the limitations of each mobile operating system with respect to privacy, security, confidentiality, integrity etc., the applications are limited within their operating system or device itself. Hence the inter-application communication is very limited in Android, iOS, Windows Phone etc. as in [16] in a home network.

\section{PRototype ImPlementation}

There are several types of applications like games, social media, entertainment etc. installed on smart devices like Smartphone, Tablet, Notebook, Smart TV etc. These applications are very limited to share their data with other applications on other devices in a home network. If interapplication communication feature is implemented on an application, then an application on a device can discover, connect and share data with other application on another device as in [17].

Based on the proposed architecture for inter-application communication in a home network as in [18], this prototype is implemented to demonstrate the inter-application communication in a home network. This prototype implementation includes two applications that reside on two different operating systems and devices can discover, communicate and share data with each other.

\subsection{EXAMPLE SCENARIO OF INTER-APPLICATION COMMUNICATION}

A Phone Contact Book application on a Windows 10 Notebook can send a selected contact's phone number to a Phone Dialer application on an Android Smartphone to call that contact in a home network as in [Figure 1].

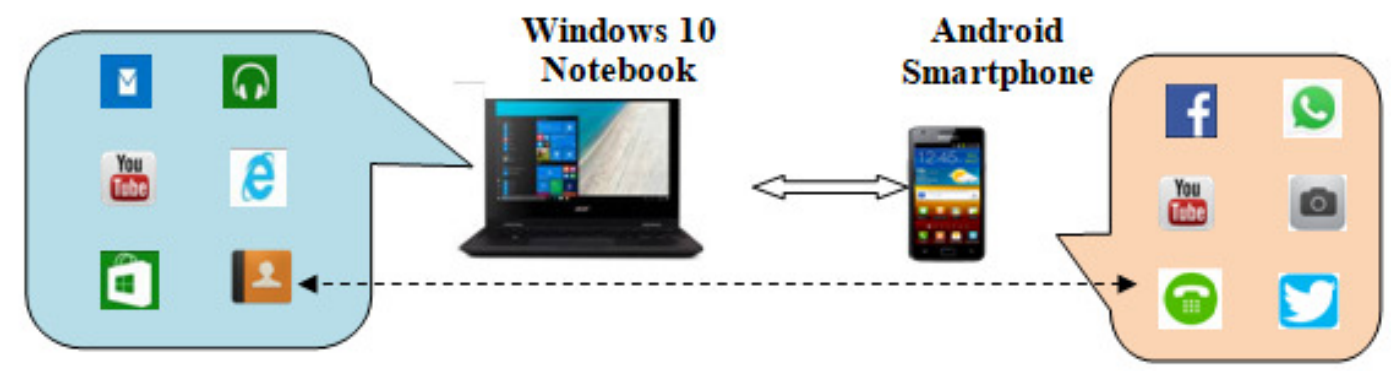

Figure 1. Inter-application communication between a My Contact Book and My Phone Dialer application on two devices in a home network

\subsection{IMPLEMENTATION ENVIRONMENT}

This prototype implementation includes two applications: My Contact Book and My Phone Dialer. The My Contact Book is a desktop application to store the details of contacts like name and phone number. My Contact Book app is based on Microsoft Windows 10 operating system. 
The My Phone Dialer is a mobile application to make phone call to a contact. My Phone Dialer app is based on Android 9 operating system.

\subsection{Process Flow Of ApPlications}

The My Contact Book app to be installed on a Notebook with Windows 10 operating system and the My Phone Dialer app to be installed on a Smartphone with Android 9 operating system. The process flow of the prototype implementation as in [Figure 2, 3, 4] is described in detail here.

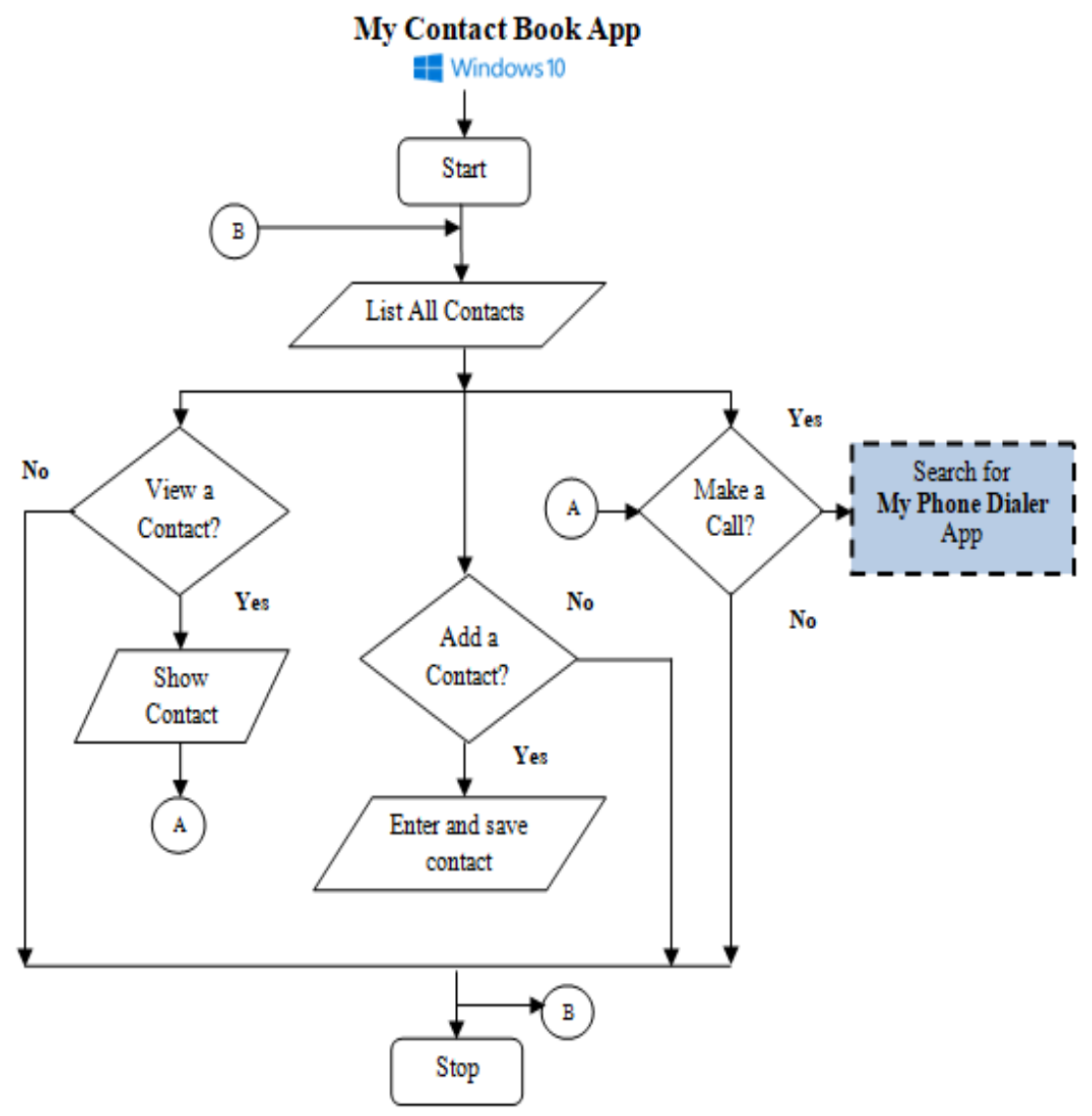

Figure 2. Process flow of My Contact Book App on a Windows 10 Notebook in a home network 


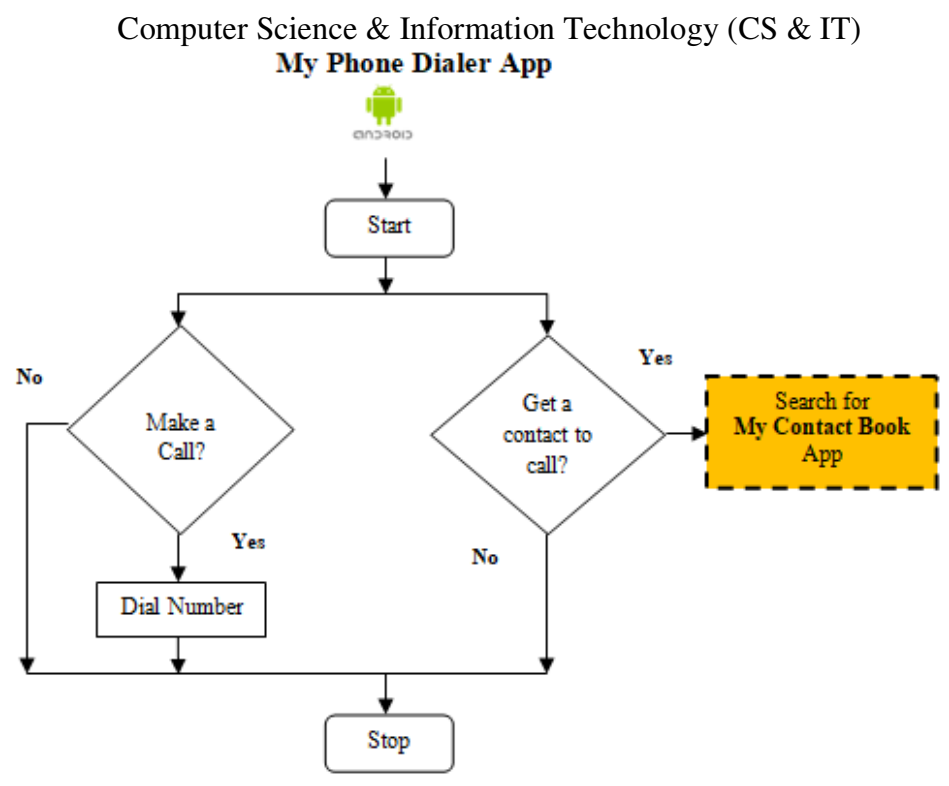

Figure 3. Process flow of My Phone Dialer App on an Android Smartphone in a home network

\section{My Contact Book}

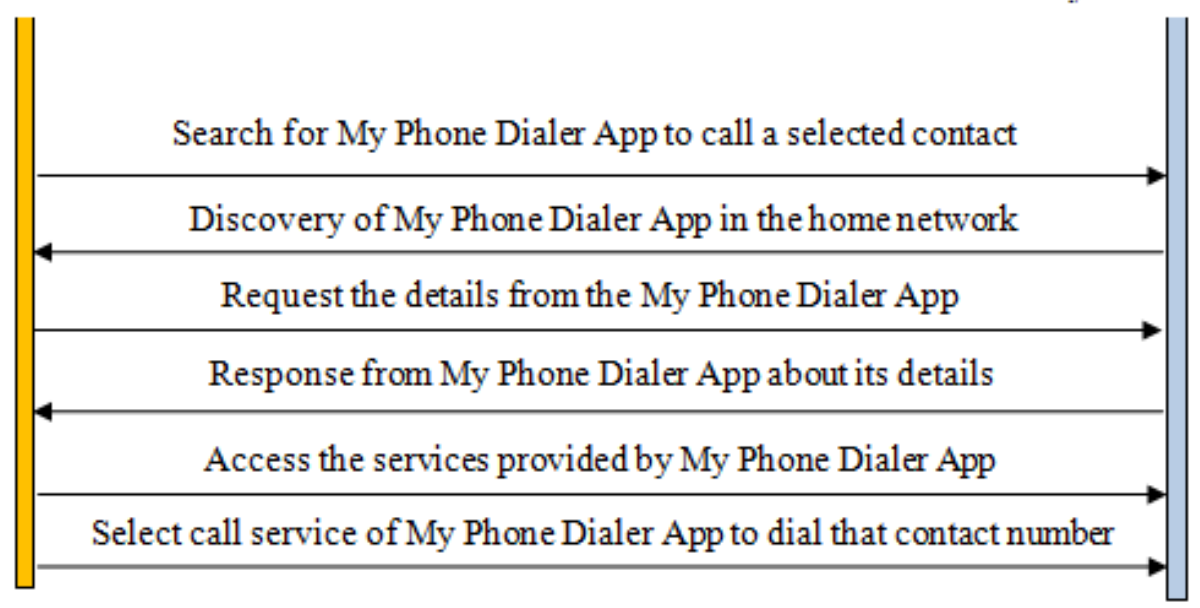

Figure 4. Inter-application communication flow between the My Contact Book App and My Phone Dialer App in a home network

When My Contact Book app is started on the Windows Notebook, new contacts can be added and the details of already existing contacts can be viewed by the user. Once a contact is selected, then the My contact Book app searches for the availability of My Phone Dialer app in the home network to call that contact. If the My Phone Dialer app is not started on an Android Smartphone, then it is not discovered by the My Contact Book app. When the My Phone Dialer app is started on the Smartphone by the user, then it is discovered by the My Contact Book app. Once if it is identified, then the user can view the details of the My Phone Dialer app like app name, app developer, version and its services ("call" service). Once the user selected the "call" service, then My Phone Dialer app automatically dials the phone number of the selected contact on Smartphone. When My Phone Dialer app is stopped or closed, then it is not discovered by the My Contact Book app and vice versa in the home network. 
The availability of both applications on these devices is instantly identified by the user in the home network through the application discovery feature embedded in these two applications. The inter-application communication is implemented in this prototype whereby these two applications can discover, connect and share data with each other despite of their underlying operating system and device type.

\subsection{USER INTERFACE OF APPLiCATIONS}

The user interface of the My Contact Book app as in [Figure 5-8, 11-12] and My Phone Dialer app as in [Figure 9, 10, 13-20] provides the flow of communication between these two applications in the home network.

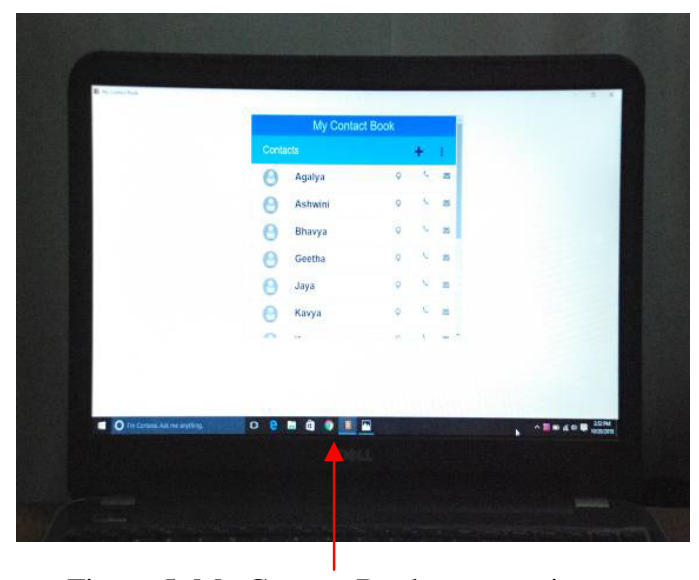

Figure 5. My Contact Book app running on

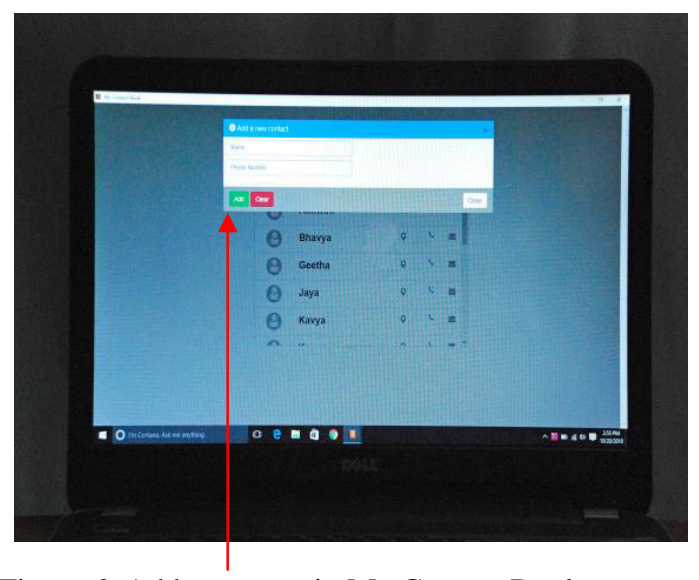

Figure 6. Add a contact in My Contact Book app Windows 10 Notebook

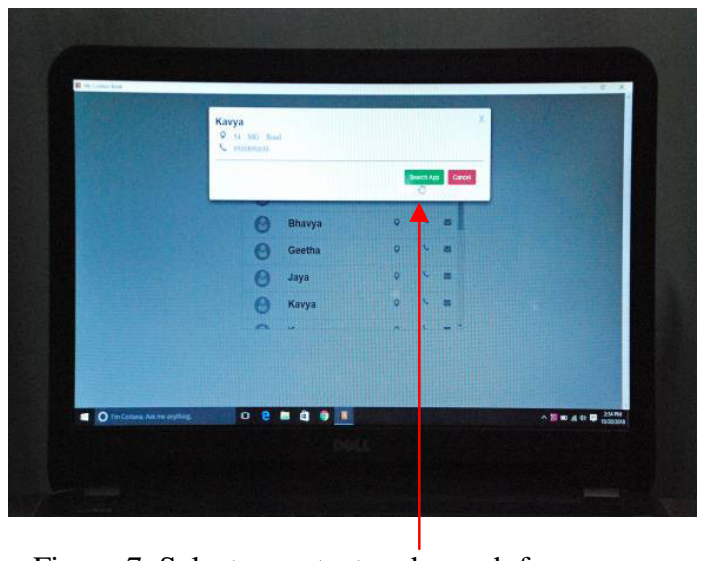

Figure 7. Select a contact and search for a Dialer app to make a call

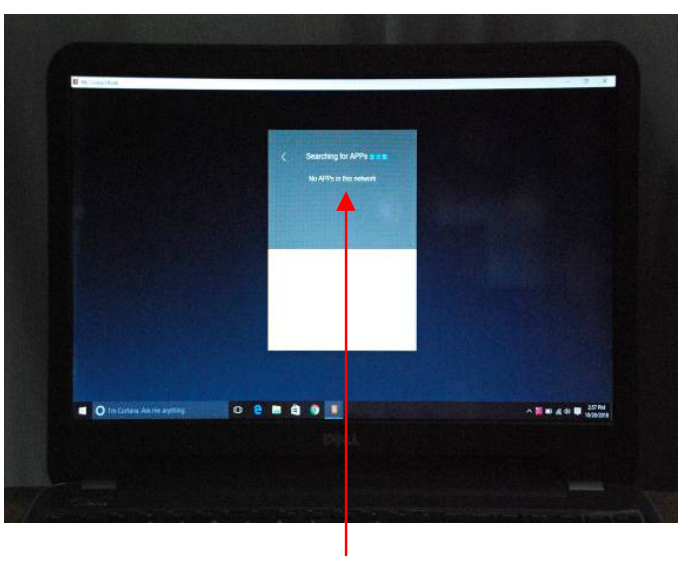

Figure 8. No applications available in the home network

(if My Phone Dialer app not started on Smartphone) 


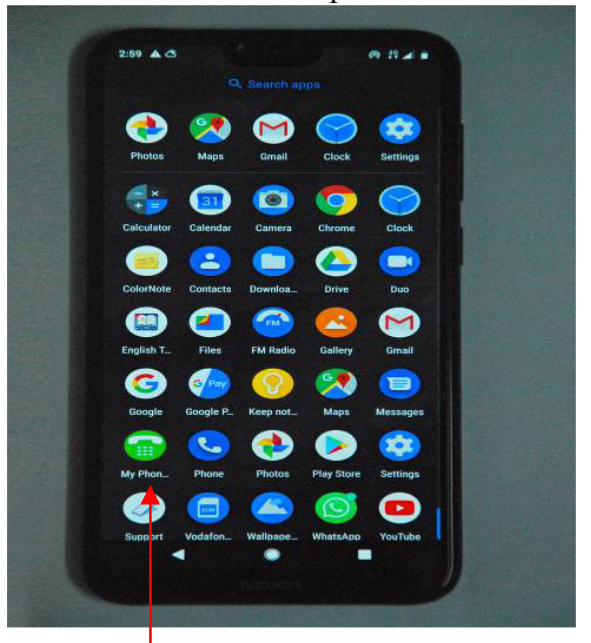

Figure 9. My Phone Dialer app installed on an Android Smartphone

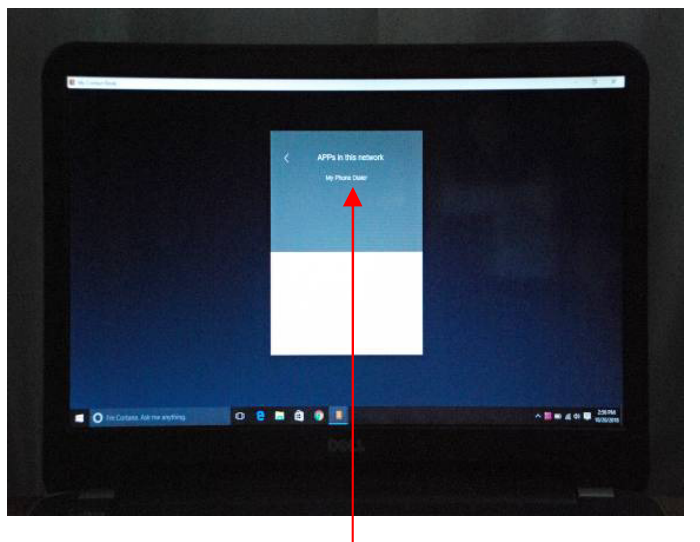

Figure 11. My Phone Dialer app is available in the home network

(if My Phone Dialer app started on Android Smartphone)

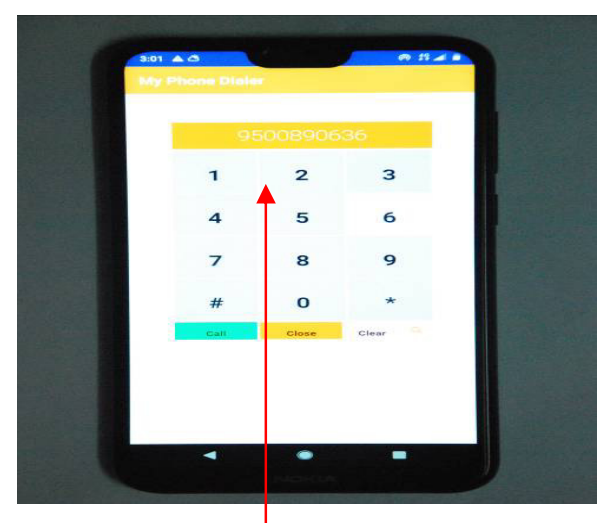

Figure 13. My Phone Dialer app receives the selected phone number from My Contact Book app

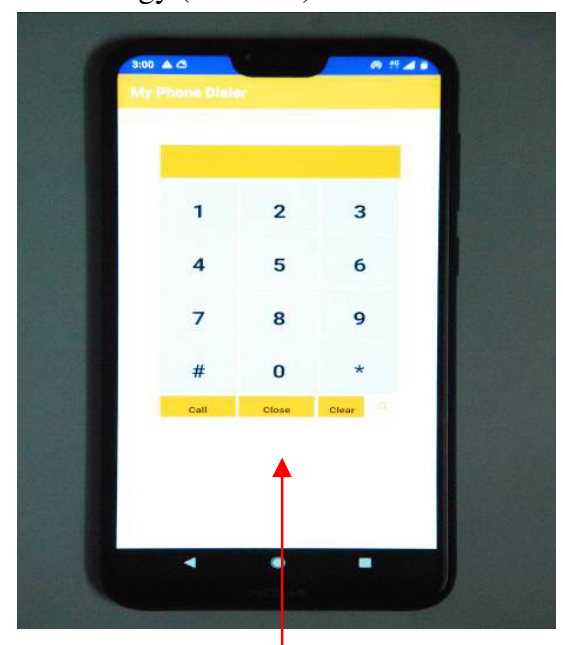

Figure 10. My Phone Dialer app running on an Android Smartphone

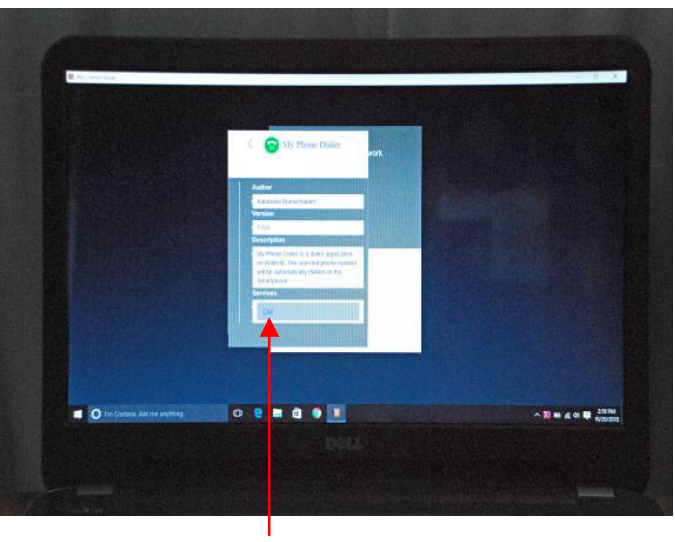

Figure 12. User views the details of My Phone Dialer app along with its services and selects the call service

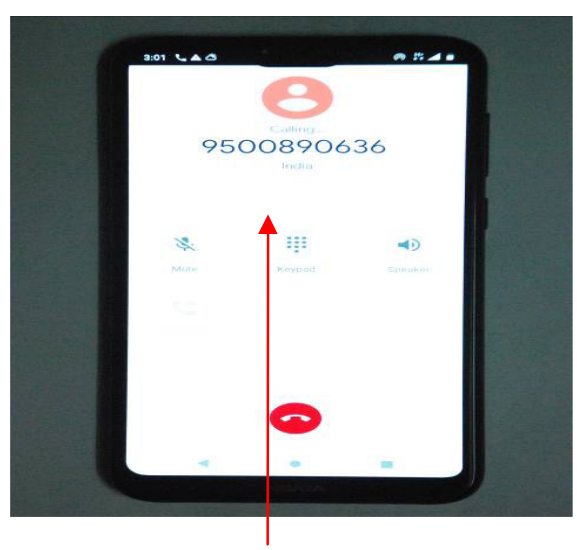

Figure 14. My Phone Dialer app automatically dials the phone number of the selected contact 


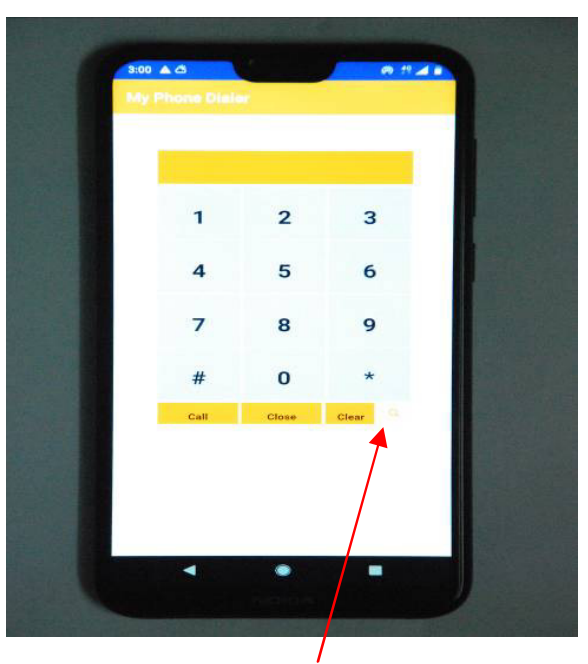

Figure 15. User searches for My Contact Book app in the home network

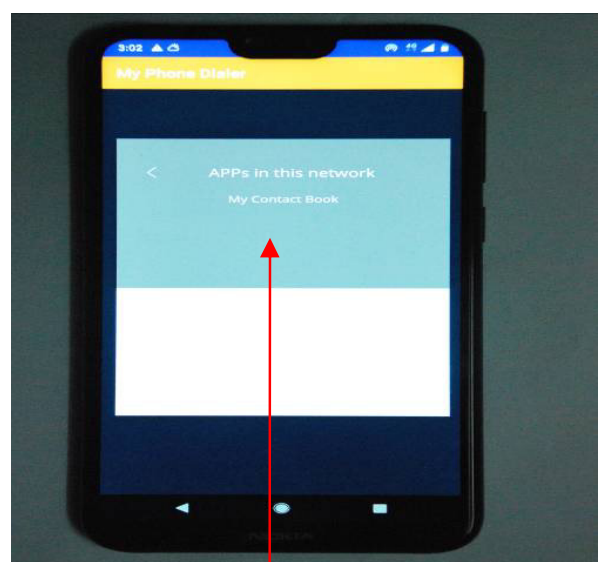

Figure 17. My Contact Book app is available in the home network

(if My Contact Book app started on Windows PC)

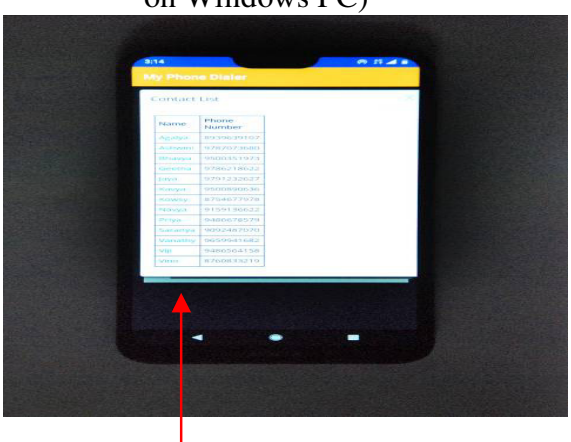

Figure 19. User views the contacts from My Contact Book app and selects a contact to make a call

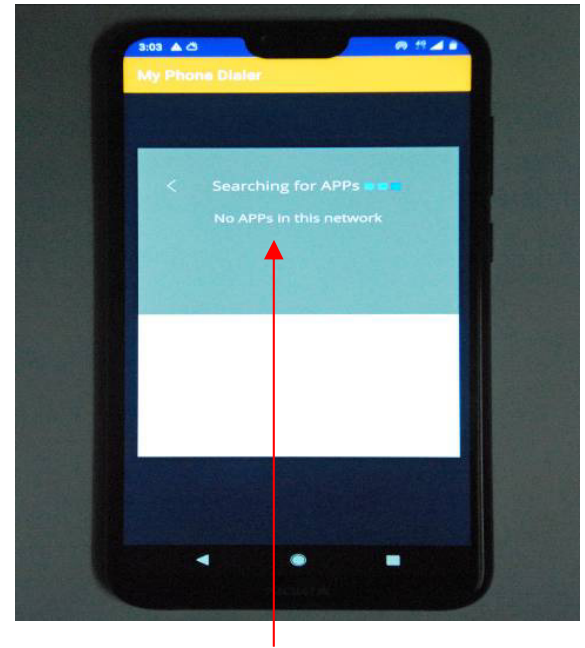

Figure 16. No applications available in the home network

(if My Contact Book app not started on Windows PC)

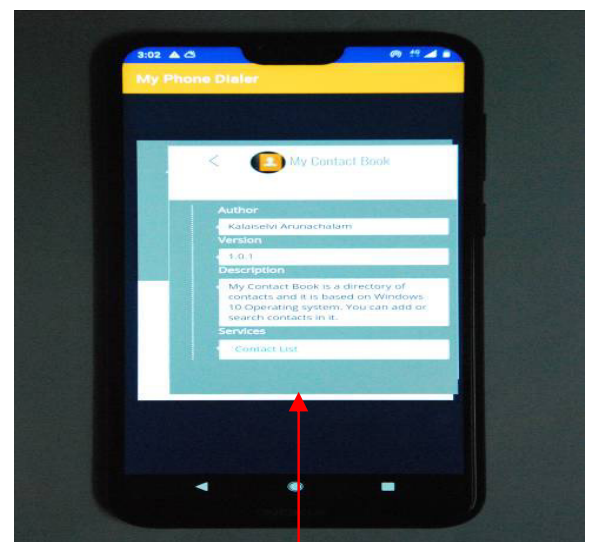

Figure 18. User views the details of My Contact Book app along with its services

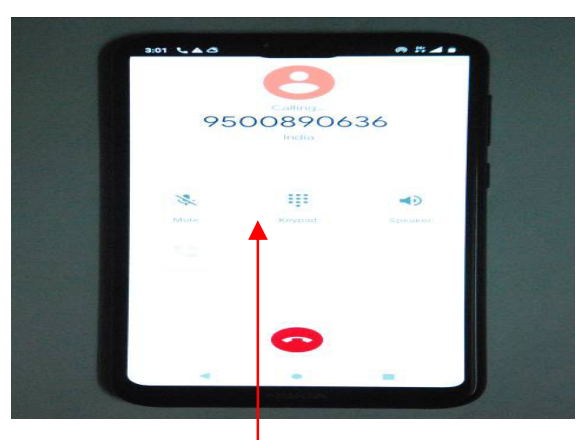

Figure 20. My Phone Dialer app dials the phone number of the selected contact 


\subsection{Evaluation}

The My Contact Book app is installed on a Notebook with Windows 10 operating system, 1.5 GHz Intel processor, 2GB RAM and 500 GB hard disk. The My Phone Dialer app is installed on a Nokia 6.1 Plus Smartphone with Android 9 Pie operating system, Qualcomm Snapdragon 636 processor, 4GB RAM and 64GB internal memory. Both applications were tested on these devices in a home network and both of them worked well as expected by discovering each of their presence, communicating with each other by accessing their services and by invoking action on each other in the home network.

Some of the application attributes like app name, app developer name, version, description and services are used by these applications in their app description as in [18]. These two applications were developed based on a sample scenario to demonstrate the inter-application communication in the home network. There are numerous scenarios available where by any kind of applications can discover, communicate and share their data with other applications in a home network.

\section{CONCLUSION}

The popularity of smart devices and the heterogeneity of applications used on these devices with multitude of data that serves the users around the world in their digital living environment. The data sharing between the applications across these heterogeneous devices without using any intermediary hardware or software would be beneficial to the users in a home network. These applications use various types of data which can be accessed and shared by the users through inter-application communication. Hence it is implemented in a home network with a prototype in which two different applications on two different device types and operating systems can discover, communicate and share data with each other. Following the implementation, the prototype is evaluated by testing it in a home network and it worked well as expected.

\section{REFERENCES}

[1] Kenny Fok, Jihyun Hwang, Eric Chi Chung Yip, Mikhail A. Lushin, (2012) "Providing secure interapplication communication for a mobile operating environment", United States Patent.

[2] Venu Ambekar, Robert Neff, John Zaleski, (2005) "Executable application interoperability and interface creation system", United States Patent.

[3] Samir Nigam, (2014) "Deploy anywhere framework for heterogeneous mobile application development", United States Patent.

[4] Sachin Desai, Qingqing Liu, Ronald Fischer, (2016) "Method and system for secured interapplication communication in mobile devices", United States Patent.

[5] Lloyd Leon Burch, Baha Masoud, (2018) "Audio proximity-based mobile device data sharing", United States Patent.

[6] Hongwei Guo, Long He, Fangming Li, Xiao Qiu Tang, Xi Ning Wang, Jing Zhang, (2018) "Data sharing between multiple applications running on a mobile device", United States Patent.

[7] Kyungjin Kim, Kiwon Lee, Sungil Cho, Jiyoung Hong, Sungeun Kim, (2013) "Data sharing between smart devices", United States Patent.

[8] Thomas Edward Wagner, Robert Elliott Whiteman, (2014) "Secure App-To-App Communication", United States Patent. 
36 Computer Science \& Information Technology (CS \& IT)

[9] Andrew Mark Earnshaw, Jianfeng Weng, (2017) "System and method for sharing data across multiple electronic devices", United States Patent.

[10] Stefan Poslad, (2009) Ubiquitous Computing: Smart Devices, Environments and Interactions, Queen Mary, University of London, United Kingdom.

[11] Google Play: https://play.google.com/store

[12] App Store (iOS): https://apple.com/appstore

[13] Microsoft Store: https://www.microsoft.com/store/apps

[14] BlackBerry World: http://appworld.blackberry.com/

[15] Number of apps in leading app stores $2018 \quad$ । Statista: https://www.statista.com/statistics/276623/number-of-apps-available-in-leading-app-stores/

[16] Kalaiselvi Arunachalam, Dr. Gopinath Ganapathy, (2015) "The Comparison of Inter-Application Communication Mechanisms in Mobile Operating Systems", International Journal of Computer Science and Mobile Applications (IJCSMA), Vol. 3. Issue. 10, pp. 48-58.

[17] Kalaiselvi Arunachalam, Dr. Gopinath Ganapathy, (2016) "Discovery and Identification of an Application for Inter-Application Communication on a Home Network Using UPnP", Journal of Computers (JCP), Vol. 11, No. 6, pp. 488-496.

[18] Kalaiselvi Arunachalam, Dr. Gopinath Ganapathy, (2017) "Extending UPnP for Application Interoperability in a Home Network", International Journal of Electrical and Computer Engineering (IJECE), Vol. 7, No. 4, pp. 2085-2093.

\section{Authors}

Kalaiselvi Arunachalam received the B.Sc. degree in Physics from the University of Madras, India and M.C.A degree in Computer Applications from the Anna University, India. She is currently a Ph.D. scholar in the School of Computer Science Engineering and Applications, Bharathidasan University, India. Her research interests include Home Networking, Communication Software and Systems.

Dr. Gopinath Ganapathy received the B.Sc. degree in Computer Science from the Bharathidasan University, India, M.C.A degree in Computer Applications from the St. Joseph's College Autonomous, India and Ph.D from the Madurai Kamaraj University, India. He is currently the Registrar, Bharathidasan University, India. His research interests include Semantic Web, NLP, Ontology, and Text Mining.
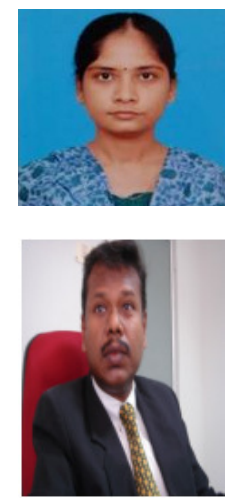